PROCEEDINGS OF THE

AMERICAN MATHEMATICAL SOCIETY

Volume 127, Number 4, April 1999, Pages 985-991

S 0002-9939(99)05149-7

\title{
A SPACE ON WHICH DIAMETER-TYPE PACKING MEASURE IS NOT BOREL REGULAR
}

\author{
H. JOYCE
}

(Communicated by Christopher Croke)

\begin{abstract}
We construct a separable metric space on which 1-dimensional diameter-type packing measure is not Borel regular.
\end{abstract}

\section{INTRODUCTION}

In [11] Taylor and Tricot introduced a new measure on $\mathbf{R}^{n}$, which they named packing measure. This measure was intended as a type of dual to Hausdorff measure, where the idea of economical coverings by sets of small diameter was replaced by that of extravagant packings by balls of small diameter.

For clarity in what follows we now provide a definition of what we shall refer to as diameter-type packing measure. Our notation differs from that of [11], and we do not restrict our attention to $\mathbf{R}^{n}$. We also follow recent practice (see $[3,4,5,6]$, and $[9,5.10])$ in using closed balls rather than open in our definition of packing measure.

By a packing of a subset $S$ of a metric space $X$ we mean a finite or countable collection of closed balls $\left\{B\left(x_{i}, r_{i}\right): x_{i} \in S\right\}$ such that for each $i \neq j$,

$$
B\left(x_{i}, r_{i}\right) \cap B\left(x_{j}, r_{j}\right)=\emptyset .
$$

A $\delta$-packing is a packing such that for each $i$, diam $B\left(x_{i}, r_{i}\right) \leq \delta$.

If $h$ is a Hausdorff function, that is, a non-decreasing function from $\mathbf{R}^{+}$to $\mathbf{R}^{+}$ with $h(0+)=0$, then $\mathcal{P}^{h}(S)$, the diameter-type h-packing measure of $S$, may be defined thus:

$$
\begin{aligned}
& P_{\delta}^{h}(S)=\sup \left\{\sum h\left(\operatorname{diam} B\left(x_{i}, r_{i}\right)\right):\left\{B\left(x_{i}, r_{i}\right)\right\} \text { a } \delta \text {-packing of } S\right\}, \\
& P_{0}^{h}(S)=\lim _{\delta \rightarrow 0} P_{\delta}^{h}(S), \\
& \mathcal{P}^{h}(S)=\inf \left\{\sum_{1}^{\infty} P_{0}^{h}\left(S_{i}\right): S \subset \bigcup_{1}^{\infty} S_{i}\right\} .
\end{aligned}
$$

In [4] it was noted that this definition led to a problem, namely that one could not be sure that packing measure, thus defined, was Borel regular, that is, that

Received by the editors December 11, 1996.

1991 Mathematics Subject Classification. Primary 28A75.

Key words and phrases. Packing measure, Borel regularity.

(C)1999 American Mathematical Society 
every set was contained inside a Borel set of equal measure. A possible solution proposed there was to modify this definition by replacing the final line by

$$
\mathcal{P}^{h}(S)=\inf \left\{\sum_{1}^{\infty} P_{0}^{h}\left(S_{i}\right): S \subset \bigcup_{1}^{\infty} S_{i} \text {, and } S_{i} \text { Borel }\right\} .
$$

Haase also introduced radius-type packing measure (see [2]), where he replaced $\operatorname{diam} B\left(x_{i}, r_{i}\right)$ by $2 r_{i}$ throughout Taylor and Tricot's definition. We write $R_{\delta}^{h}(S)$, $R_{0}^{h}(S)$ and $\mathcal{R}^{h}(S)$ for the radius-type analogues of $P_{\delta}^{h}(S), P_{0}^{h}(S)$ and $\mathcal{P}^{h}(S)$, and $\mathcal{P}^{s}$ and $\mathcal{R}^{s}$ for the measures resulting from the Hausdorff functions $h(r)=r^{s}$.

Radius-type packing measures are now often used. However, Haase's suggested modification to the definition of diameter-type packing measure has not entered common use. This may be because the problem of non-Borel regularity does not arise on Euclidean spaces, provided $h$ is left-continuous (see Theorem 1.2 below). Also, a useful (and immediate) fact about packing measures is that $\mathcal{P}^{h}(S) \leq P_{0}^{h}(S)$ for every set $S$; if we modify packing measure as suggested above, then there is no longer any reason why this inequality should hold.

In this paper we give a construction which shows that if closed balls are used instead of open in the definition of diameter-type packing measures, then some such modification is indeed needed in order to ensure Borel regularity on non-Euclidean spaces.

The added step in the definition of packing measures as compared to Hausdorff measures makes our result seem perhaps a little less surprising. For example, in [10] the question of the measurability of the packing and Hausdorff measure and dimension functions is investigated. It is shown there that while the Hausdorff dimension and measure functions are indeed measurable with respect to the Borel field generated by the compact subsets of a separable metric space, the packing measure and dimension functions are not.

Lemma 1.1. Let $X$ be a metric space and let $S \subseteq X$. If $h$ is a left-continuous Hausdorff function, then

If also $X=\mathbf{R}^{n}$, then

$$
R_{0}^{h}(S)=R_{0}^{h}(\text { Clos } S)
$$

$$
P_{0}^{h}(S)=P_{0}^{h}(\operatorname{Clos} S)
$$

The proof is easy.

Theorem 1.2. Let $X$ be a metric space and let $S \subseteq X$. If $h$ is a left-continuous Hausdorff function, then $\mathcal{R}^{h}$ is a Borel regular measure. If also $X=\mathbf{R}^{n}$, then $\mathcal{P}^{h}$ is a Borel regular measure.

Proof. Lemma 1.1 ensures that, under these assumptions, each set is contained in an $F_{\sigma \delta}$ set of the same measure (see for example $[9,5.10]$ ).

However, the statement that the pre-measure $P_{0}^{h}$ of each set is the same as that of its closure is not necessarily true, even for $h$ continuous, on spaces other than $\mathbf{R}^{n}$; an example was provided in $[7,1.4 .4]$. This note takes the idea of that example, and extends it to provide a construction of a separable metric space with a non-Borel subset of $\mathcal{P}^{1}$ measure zero, such that every Borel subset of the metric space containing that set has infinite $\mathcal{P}^{1}$ measure. In this construction, the highly discrete metric will allow us to control the number of disjoint balls which may be centred in certain subsets of our metric space. 


\section{The CONSTRUCTION}

Our metric space $X$ is constructed as follows.

First we construct a code space $Y$ with a discrete metric. The measure $\mu$ on $Y$, which is the image of $s$-dimensional Hausdorff measure on the middle third Cantor set $C(s=\log 2 / \log 3)$ under the homeomorphism via the natural coding of $C$, will play an auxiliary role.

In Lemma 2.1 we find a non-Borel subset $S$ of $Y$. We then take the next step in building up our metric space $X$ by adding to $Y$ countably many copies $Z^{k}$ of $Y \backslash S$ and suitably extending our metric. Our aim here is to extend the metric space $Y$ to a space where the 1-dimensional packing measure of $S$ is zero, but that of any Borel set containing $S$ is infinite. The role of these sets $Z^{k}$ is to ensure that balls of certain radii centred in $Y \backslash S$ have larger diameter than balls of the same radii centred in $S$ itself. The final step in constructing $X$ will then be to add families of sequences to our metric space and to extend our metric once more. The purpose of these sequences is to ensure that there are not too many disjoint balls of certain diameters centred in $S$.

In Lemma 2.2 we see that if a subset $V$ of $Y \backslash S$ has positive $\mu$-measure, then it must satisfy $P_{0}^{1}(V)=\infty$. In Lemma 2.1 it was established that if $B$ is a Borel subset of $Y$ containing $S$, and $B \subseteq \bigcup_{i} B_{i}$, then one of the $B_{i}$ 's must contain such a set $V$. In Lemmas 2.3 and 2.4 we see that a Borel subset $B$ of $X$ which contains $S$ must have infinite $\mathcal{P}^{1}$ measure, and that $S$ itself has zero $\mathcal{P}^{1}$ measure. These two lemmas lead directly to our main result, which is Theorem 2.5.

As the first step in our construction, we choose a positive sequence $\left(d_{j}\right)_{j}$ with $d_{j+1} \leq d_{j} / 2$, and an increasing sequence $\left(m_{j}\right)_{j}$ of integers, and write $n_{j}=2^{m_{j}}$, $N_{j}=\prod_{i=1}^{j} n_{i}$, and $M_{j}=\sum_{i=1}^{j} m_{i}\left(\right.$ so $\left.N_{j}=2^{M_{j}}\right)$, such that

$$
\begin{gathered}
\sum_{i=1}^{\infty} N_{k} d_{k+1}<\infty, \\
\lim _{k \rightarrow \infty} N_{k} d_{k}=\infty .
\end{gathered}
$$

To see that this is possible choose $d_{1}>0$ and a positive integer $m_{1}$. Suppose $d_{1}, \ldots, d_{k}$ and $m_{1}, \ldots, m_{k}$ have been chosen. Then we can choose $d_{k+1} \leq d_{k} / 2$ sufficiently small such that $N_{k} d_{k+1}<2^{-(k+1)}$, and $m_{k+1}$ sufficiently large such that $N_{k+1} d_{k+1}>k+1$.

We write

$$
Y=\left\{\left(i_{1}, i_{2}, \ldots\right): 1 \leq i_{j} \leq n_{j} \text { for each } j \geq 1\right\}
$$

with

$$
\operatorname{dist}((i),(j))=2 d_{k},
$$

where $k$ is least such that $i_{k} \neq j_{k}$. We shall write

$$
Y_{i_{1}, \ldots, i_{l}}=\left\{\left(i_{1}, i_{2}, \ldots, i_{l}, j_{l+1}, j_{l+2}, \ldots\right): 1 \leq j_{l+q} \leq n_{l+q} \text { for each } q \geq 1\right\},
$$

and refer to such sets as cylinder sets of $Y$. We note that $Y$ is homeomorphic to the middle third Cantor set $C$. To see this, for each $k \geq 1$ write $\varphi_{k, 1}, \ldots, \varphi_{k, n_{k}}$ for the $n_{k}=2^{m_{k}}$ similitudes from $C$ onto the cylinder sets of $C$ of diameter $3^{-m_{k}}$. We may then define a homeomorphism from $Y$ to $C$ by $f\left(\left(i_{1}, i_{2}, \ldots\right)\right)=\bigcap_{k=1}^{\infty} \varphi_{1, i_{1}} \circ \ldots \circ$ 
$\varphi_{k, i_{k}}(C)$. We shall use this homeomorphism to define a (Borel regular) measure $\mu$ on $Y$ by setting

$$
\mu(S)=\mathcal{H}^{s}(f(S)) \text { for each } S \subseteq Y,
$$

where $s=\log 2 / \log 3$.

Lemma 2.1. There is a non-Borel set $S \subset Y$ such that if $B$ is a Borel set with $S \subset B \subset Y$, and $\left\{B_{i}\right\}$ are subsets of $Y$ with $B \subset \bigcup_{i=1}^{\infty} B_{i}$, then there is an $i$ with $\mu\left(B_{i} \backslash S\right)>0$.

Proof. Since $Y$ is a complete metric space without isolated points, and since $0<$ $\mu(Y)<\infty$, we may find $S \subset Y$ such that if $K$ is a closed subset of $Y$ with $\mu(K)>0$, then $K$ intersects both $S$ and its complement (see [1, 2.2.4]). This property ensures that for each such $K, K \cap S$ is not $\mu$-measurable, and in particular, since Borel subsets of $Y$ are $\mu$-measurable, that $S$ is not a Borel subset of $Y$.

If $B$ is a Borel subset of $Y$ containing $S$, then $B$ contains some closed set $D$ such that $\mu(D)>0$ (otherwise $B$, like $S$, would not be $\mu$-measurable). Then $\mu(D \backslash S)>0$, otherwise $D \backslash S$, and therefore $D \cap S$, would be $\mu$-measurable, contradicting the choice of $S$. If $B \subset \bigcup_{i=1}^{\infty} B_{i}$, we have $D \backslash S \subset \bigcup_{i=1}^{\infty}\left(B_{i} \backslash S\right)$, so there is an $i$ with $\mu\left(B_{i} \backslash S\right)>0$, proving the lemma.

Choose $S \subset Y$ according to Lemma 2.1. Let $Z=Y \backslash S$. For each $k \geq 1$, let

$$
Z^{k}=Z \times\left\{d_{k}\right\}
$$

As for $Y$, we write

$$
Z_{i_{1}, \ldots, i_{l}}^{k}=\left\{\left(\left(i_{1}, i_{2}, \ldots, i_{l}, j_{l+1}, j_{l+2}, \ldots\right), d_{k}\right): 1 \leq j_{l+q} \leq n_{l+q} \text { for each } q \geq 1\right\} .
$$

We extend the metric on $Y$ to a metric on $Y \cup \bigcup_{k=1}^{\infty} Z^{k}$ by putting

$$
\begin{aligned}
\operatorname{dist}\left(\left(x, d_{k}\right), y\right) & =\operatorname{dist}(x, y)+d_{k}, \\
\operatorname{dist}\left(\left(x, d_{k}\right),\left(z, d_{j}\right)\right) & =\operatorname{dist}(x, z)+\left|d_{k}-d_{j}\right|,
\end{aligned}
$$

for each $y \in Y, x, z \in Z$, and $k, j \geq 1$. Note that these definitions ensure that if $y \in$ $Z$, then $B\left(y, d_{k}\right) \cap Z_{i_{1}, \ldots, i_{k}}^{k}$ contains the single point $\left(y, d_{k}\right)$, and diam $B\left(y, d_{k}\right) \geq d_{k}$. However, if $y \in S$, then $B\left(y, d_{k}\right) \cap Z^{k}=\emptyset$, so a ball centred in $S$ which intersects $Z^{k}$ (and hence has diameter at least $d_{k}$ ) must have radius strictly greater than $d_{k}$.

Recall that our goal is to extend $Y$ to a space where the 1-dimensional packing measure of $S$ is zero, but that of any Borel set containing $S$ is infinite. The final step, therefore, will be to add to our metric space sequences of points whose distances from points in $Y_{i_{1}, \ldots, i_{k-1}}$ approach $d_{k}$; this will ensure that any two balls of radius strictly greater than $d_{k}$ centred in $Y_{i_{1}, \ldots, i_{k-1}}$ must intersect each other, but will have no effect on balls of radius $d_{k}$ or less. Distances from points in these sequences to each other, and to points outside $Y_{i_{1}, \ldots, i_{k-1}}$, will be chosen simply to satisfy the triangle inequality.

We now complete the construction. Let $Q^{0}=\left\{q_{j}^{0}: j \geq 1\right\}$ satisfy

$$
\begin{aligned}
\operatorname{dist}\left(q_{j}^{0}, q_{k}^{0}\right) & =d_{1} / 2, \\
\operatorname{dist}\left(q_{j}^{0}, y\right) & =\left(1+2^{-j}\right) d_{1} \text { for each } y \text { in } Y, \\
\operatorname{dist}\left(q_{j}^{0}, z\right) & =\left(1+2^{-j}\right) d_{1}+d_{k} \text { for each } z \text { in } Z^{k} .
\end{aligned}
$$


For each $\left(i_{1}, \ldots, i_{k}\right)$ we also add a sequence $Q^{i_{1}, \ldots, i_{k}}=\left\{q_{j}^{i_{1}, \ldots, i_{k}}: j \geq 1\right\}$ such that for $p \geq 1, r \neq l$ and $\left(i_{1}, \ldots, i_{k}\right) \neq\left(j_{1}, \ldots, j_{k}\right)$,

$$
\begin{aligned}
& \operatorname{dist}\left(q_{r}^{i_{1}, \ldots, i_{k}}, q_{l}^{i_{1}, \ldots, i_{k}}\right)=d_{k+1} / 2, \\
& \operatorname{dist}\left(q_{r}^{i_{1}, \ldots, i_{k}}, q_{p}^{j_{1}, \ldots, j_{k}}\right)=\operatorname{dist}\left(Y_{i_{1}, \ldots, i_{k}}, Y_{j_{1}, \ldots, j_{k}}\right), \\
& \operatorname{dist}\left(q_{r}^{i_{1}, \ldots, i_{k}}, q_{p}^{0}\right)
\end{aligned}
$$

and for $k \neq s$,

$$
\operatorname{dist}\left(q_{r}^{i_{1}, \ldots, i_{k}}, q_{p}^{j_{1}, \ldots, j_{s}}\right)=\left|d_{k+1}-d_{s+1}\right|+\operatorname{dist}\left(Y_{i_{1}, \ldots, i_{k}}, Y_{j_{1}, \ldots, j_{s}}\right) .
$$

For each $y \in Y_{i_{1}, \ldots, i_{k}}, z \in Z_{i_{1}, \ldots, i_{k}}^{l}$ and $\left(i_{1}, \ldots, i_{k}\right) \neq\left(j_{1}, \ldots, j_{k}\right)$, we also set

$$
\begin{aligned}
\operatorname{dist}\left(q_{r}^{i_{1}, \ldots, i_{k}}, y\right) & =\left(1+2^{-r}\right) d_{k+1}, \\
\operatorname{dist}\left(q_{r}^{j_{1}, \ldots, j_{k}}, y\right) & =\left(1+2^{-r}\right) d_{k+1}+\operatorname{dist}\left(Y_{i_{1}, \ldots, i_{k}}, Y_{j_{1}, \ldots, j_{k}}\right), \\
\operatorname{dist}\left(q_{r}^{i_{1}, \ldots, i_{k}}, z\right) & =\left(1+2^{-r}\right) d_{k+1}+d_{l}, \\
\operatorname{dist}\left(q_{r}^{j_{1}, \ldots, j_{k}}, z\right) & =\left(1+2^{-r}\right) d_{k+1}+\operatorname{dist}\left(Y_{i_{1}, \ldots, i_{k}}, Y_{j_{1}, \ldots, j_{k}}\right)+d_{l} .
\end{aligned}
$$

Our final separable metric space $X$ will then be

$$
X=Y \cup \bigcup_{i=1}^{\infty} Z^{k} \cup Q^{0} \cup \bigcup_{k \geq 1}\left\{Q^{i_{1}, \ldots, i_{k}}: 1 \leq i_{j} \leq n_{j} \text { for each } 1 \leq j \leq k\right\} .
$$

Lemma 2.2. If $V \subseteq Z$ satisfies $\mu(V)>0$, then

$$
P_{0}^{1}(V)=\infty \text {. }
$$

Proof. Let $\mu(V)=a$. Let $\eta_{k}$ be the number of cylinder sets of the Cantor middle third set $C$ of diameter $3^{-M_{k}}$ needed to cover $f(V)$ (there is a total of $N_{k}=2^{M_{k}}$ such intervals). Then for $k$ sufficiently large (recalling the definition of $\mu$ and that $s=\log 2 / \log 3)$,

$$
\begin{aligned}
\eta_{k}\left(3^{-M_{k}}\right)^{s} & \geq a / 2 \\
\eta_{k} 2^{-M_{k}} & \geq a / 2 \\
\eta_{k} & \geq a N_{k} / 2 .
\end{aligned}
$$

For each $k$,

$$
\eta_{k}=\#\left\{\left(i_{1}, \ldots, i_{k}\right): Y_{i_{1}, \ldots, i_{k}} \cap V \neq \emptyset, 1 \leq i_{j} \leq n_{j}, 1 \leq j \leq k\right\} .
$$

Thus we may be assured that for $k$ large enough, there is a fixed proportion of the $N_{k}$ cylinder sets $Y_{i_{1}, \ldots, i_{k}}$ in which we may centre a ball of a packing of $V$. This ensures that in packing $V$ we may put disjoint balls of radius $d_{k}$ centred in each of $\eta_{k}$ distinct cylinder sets $Y_{i_{1}, \ldots, i_{k}}$. We now check that these balls are indeed disjoint.

Let $\left(i_{1}, i_{2}, \ldots\right)$ and $\left(j_{1}, j_{2}, \ldots\right)$ be points of $V$ with $i_{k} \neq j_{k}$. Then the two balls $B\left(\left(i_{1}, i_{2}, \ldots\right), d_{k}\right)$ and $B\left(\left(j_{1}, j_{2}, \ldots\right), d_{k}\right)$ cannot intersect in $Y$, since

$$
\operatorname{dist}\left(Y_{i_{1}, \ldots, i_{k}}, Y_{j_{1}, \ldots, j_{k}}\right) \geq 2 d_{k} .
$$

Nor can these two balls intersect in $\bigcup Z^{l}$, since $B\left(\left(i_{1}, i_{2}, \ldots\right), d_{k}\right) \cap \bigcup Z^{l} \subset \bigcup_{l} Z_{i_{1}, \ldots, i_{k}}^{l}$ and $\operatorname{dist}\left(Z_{i_{1}, \ldots, i_{k}}^{l}, Z_{j_{1}, \ldots, j_{k}}^{l}\right) \geq 2 d_{k}$ for each $l \geq 1$. Finally, they cannot intersect in any set $Q^{l_{1}, \ldots, l_{p}}$ since if $q_{j}^{l_{1}, \ldots, l_{p}} \in B\left(\left(i_{1}, i_{2}, \ldots\right), d_{k}\right)$, then

$$
\operatorname{dist}\left(Y_{j_{1}, \ldots, j_{k}}, q_{j}^{l_{1}, \ldots, l_{p}}\right) \geq 2 d_{k} .
$$


Suppose $B\left(x, d_{k}\right)$ is one of these balls. Since $x \in V \subset Z, B\left(x, d_{k}\right)$ contains the point $\left(x, d_{k}\right)$ of $Z_{i_{1}, \ldots, i_{k}}^{k}$, and hence has diameter at least $d_{k}$ (and, of course, no more than $2 d_{k}$ ). Therefore

$$
P_{2 d_{k}}^{1}(V) \geq \eta_{k} d_{k} \geq a N_{k} d_{k} / 2 .
$$

Since $N_{k} d_{k} \rightarrow \infty$ as $k \rightarrow \infty$ by choice of $N_{k}$ and $d_{k}$, recall (2), the result follows.

Lemma 2.3. If $B$ is a Borel subset of $X$ such that $B \supset S$, then $\mathcal{P}^{1}(B)=\infty$.

Proof. Now, $B \cap Y$ is a Borel subset of $Y$ containing $S$. Therefore, by Lemma 2.1, if $B \subseteq \bigcup_{i=1}^{\infty} B_{i}$, then there is an $i$ such that $\mu\left(\left(B_{i} \cap Y\right) \backslash S\right)>0$. Then, by Lemma 2.2 , $P_{0}^{1}\left(\left(B_{i} \cap Y\right) \backslash S\right)=\infty$. Since $P_{0}^{1}\left(B_{i}\right) \geq P_{0}^{1}\left(\left(B_{i} \cap Y\right) \backslash S\right)$, we have $\mathcal{P}^{1}(B)=\infty$.

Lemma 2.4. $\mathcal{P}^{1}(S)=0$.

Proof. Let $\left\{B\left(x_{i}, r_{i}\right)\right\}$ be a packing of $S$ in $X$. Fix $i$, let $k$ be such that $d_{k}<r_{i} \leq$ $d_{k-1}$, and suppose that $x_{i} \in S \cap Y_{j_{1}, \ldots, j_{k}}$. We now establish an upper bound on the diameter of $B\left(x_{i}, r_{i}\right)$ in terms of $d_{k}$ rather than $d_{k-1}$.

Now, since $\operatorname{dist}\left(Y_{j_{1}, \ldots, j_{k-1}}, Y \backslash Y_{j_{1}, \ldots, j_{k-1}}\right)=2 d_{k-1}$, we may be sure that $B\left(x_{i}, r_{i}\right)$ cannot intersect $Y \backslash Y_{j_{1}, \ldots, j_{k-1}}$. Similarly $B\left(x_{i}, r_{i}\right)$ cannot intersect $\left(\cup_{p \geq 1} Z^{p}\right) \backslash$ $\left(\bigcup_{p \geq k} Z_{j_{1}, \ldots, j_{k-1}}^{p}\right)$. Finally, if $p<k-1$, or $p \geq k-1$ and $\left(j_{1}, \ldots, j_{k-1}\right) \neq$ $\left(l_{1}, \ldots, l_{k-1}\right)$, then $\operatorname{dist}\left(q_{r}^{l_{1}, \ldots, l_{p}}, Y_{j_{1}, \ldots, j_{k}}\right)>d_{k-1}$. Therefore

$$
\begin{aligned}
B\left(x_{i}, r_{i}\right) \subseteq & Y_{j_{1}, \ldots, j_{k-1}} \cup \bigcup_{p \geq k} Z_{j_{1}, \ldots, j_{k-1}}^{l} \\
& \cup \bigcup_{p \geq 0}\left\{Q^{j_{1}, \ldots, j_{k-1}, J_{k}, \ldots, J_{k+p}}: 1 \leq J_{k+l} \leq n_{k+l} \text { each } 0 \leq l \leq p\right\} .
\end{aligned}
$$

Since the diameters of these three sets are bounded above by $2 d_{k}, 3 d_{k}$ and $2 d_{k}$ respectively, we see that $\operatorname{diam} B\left(x_{i}, r_{i}\right) \leq 7 d_{k}$.

We now find an upper bound on the number of balls of the packing such that $d_{k}<r_{i} \leq d_{k-1}$. Note that there can be no ball of the packing besides $B\left(x_{i}, r_{i}\right)$ with radius greater than $d_{k}$ and centre in $S \cap Y_{j_{1}, \ldots, j_{k-1}}$. This is because we may find $P \geq 1$ such that $q_{p}^{j_{1}, \ldots, j_{k-1}} \in B\left(x_{i}, r_{i}\right)$ for all $p \geq P$. Any other ball centred in $S \cap Y_{j_{1}, \ldots, j_{k-1}}$ with radius greater than $d_{k}$ must also contain some of these points. (This was the purpose of introducing the sequences $Q^{j_{1}, \ldots, j_{k-1}}$.) Therefore the total number of balls in the packing with radius in the range $\left(d_{k}, d_{k-1}\right]$ is no more than $N_{k-1}$ (one for each set $Y_{j_{1}, \ldots, j_{k-1}}$ ) and these balls have diameter no more than $7 d_{k}$. So, using (1),

$$
P_{0}^{1}(S) \leq \lim _{j \rightarrow \infty} 7 \sum_{k=j}^{\infty} N_{k-1} d_{k}=0 .
$$

The result follows.

Lemmas 2.3 and 2.4 and the fact that $\mathcal{P}^{1}(S) \leq P_{0}^{1}(S)$ immediately give us our final result:

Theorem 2.5. $\mathcal{P}^{1}$ is not Borel regular on $X$.

Note. The work above relies completely on the fact that we pack by closed balls. As noted in the introduction, this follows recent practice, however in Taylor and Tricot's original definition of packing measure in [11] (see also [12]), open balls 
were used. If the Hausdorff function $h$ is left-continuous, then packing measure using open balls is Borel regular, since with this definition it is easy to prove that the pre-packing measure $P_{0}^{h}$ is the same for a set as for its closure, and therefore that each set is contained in an $F_{\sigma \delta}$ set of the same measure. Thus the work of this paper is in the style of previous papers (see $[6,8]$ ), in which seemingly minor differences between alternative definitions of packing measure are shown to lead to significantly different measure-theoretic properties.

We also note that the choice of Hausdorff function $h(r)=r$ above was in no way crucial to the result. This choice was made partly to simplify the presentation, but mostly to emphasise the fact that the existence of a space on which diameter-type $h$-packing measure is not Borel regular in no way depends on some delicate choice of $h$. Clearly a similar space could be constructed for any Hausdorff function $h$ by modifying the choice of the distances $d_{k}$ in a suitable manner.

\section{ACKNOWLEDGEMENT}

This work was supported by EU TMR Grant, Contract number ERBFMBICT96039.

\section{REFERENCES}

[1] H. Federer, Geometric Measure Theory. Springer-Verlag, 1969. MR 41:1976

[2] H. Haase, Non- $\sigma$-finite sets for packing measure. Mathematika 33 (1986), 129-136. MR 88a:28003

[3] H. Haase, Packing measures on ultrametric spaces. Studia. Math. 91 (1988), 189-203. MR 90b:28016

[4] H. Haase, The packing theorem and packing measure. Math. Nachr. 146 (1990), 77-84. MR 91m:28006

[5] J. D. Howroyd, On Hausdorff and packing dimension of product spaces. Math. Proc. Camb. Phil. Soc. 119 (1996), 715-727. MR 96j:28006

[6] H. Joyce, Concerning the problem of subsets of finite positive packing measure. J. London Math. Soc. (2) 56 (1997), 557-566. CMP 98:06

[7] H. Joyce, Packing measures, packing dimensions, and the existence of sets of positive finite measure. Thesis, University College London, 1995.

[8] H. Joyce and D. Preiss, On the existence of subsets of finite positive packing measure. Mathematika 42 (1995), 15-24. MR 96g:28010

[9] P. Mattila, Geometry of Sets and Measures in Euclidean Spaces. Cambridge University Press, 1995.

[10] P. Mattila and R. D. Mauldin, Measure and dimension functions: measurability and densities. Math. Proc. Camb. Phil. Soc. 121 (1997), 81-100. MR 97k:28014

[11] S. J. Taylor and C. Tricot, Packing measure and its evaluation for a Brownian path. Trans. Amer. Math. Soc. 288 (1985), 679-699. MR 87a:28002

[12] C. Tricot, Two definitions of fractional dimension. Math. Proc. Camb. Phil. Soc. 91 (1982), 57-74. MR 84d:28013

Department of Mathematics, University of Jyväskylä, SF-40351 Jyväskylä, Finland

E-mail address: joyce@math.jyu.fi

Current address: 10 Shearwater, Orton Wistow, Peterborough, Cambs PE2 64W, England 